\title{
High-throughput fetal fraction amplification increases analytical performance of noninvasive prenatal screening
}

\author{
Noah C. Welker, PhD ${ }^{1}$, Albert K. Lee, PhD², Rachel A. S. Kjolby, PhD², Helen Y. Wan, BS, MBA², \\ Mark R. Theilmann, $A B^{2}$, Diana Jeon, $B A^{2}$, James D. Goldberg, $M^{2}{ }^{2}$, Kevin R. Haas, PhD $^{2}$, \\ Dale Muzzey, $\mathrm{PhD}{ }^{2}$ and Clement S. Chu, $\mathrm{PhD}^{2}$
}

Purpose: The percentage of a maternal cell-free DNA (cfDNA) sample that is fetal-derived (the fetal fraction; FF) is a key driver of the sensitivity and specificity of noninvasive prenatal screening (NIPS). On certain NIPS platforms, $>20 \%$ of women with high body mass index (and $>5 \%$ overall) receive a test failure due to low FF $(<4 \%)$.

Methods: A scalable fetal fraction amplification (FFA) technology was analytically validated on 1264 samples undergoing wholegenome sequencing (WGS)-based NIPS. All samples were tested with and without FFA.

Results: Zero samples had FF $<4 \%$ when screened with FFA, whereas 1 in 25 of these same patients had FF $<4 \%$ without FFA. The average increase in FF was 3.9-fold for samples with low FF (2.3-fold overall) and $99.8 \%$ had higher FF with FFA. For all abnormalities screened on NIPS, $z$-scores increased 2.2-fold on average in positive samples and remained unchanged in negative samples, powering an increase in NIPS sensitivity and specificity.

Conclusion: FFA transforms low-FF samples into high-FF samples. By combining FFA with WGS-based NIPS, a single round of NIPS can provide nearly all women with confident results about the broad range of potential fetal chromosomal abnormalities across the genome.

Genetics in Medicine (2021) 23:443-450; https://doi.org/10.1038/s41436020-01009-5

Keywords: fetal fraction; cell-free DNA; noninvasive prenatal screening; analytical validation; body mass index

\section{INTRODUCTION}

Since its introduction into clinical care nearly a decade ago, ${ }^{1-5}$ noninvasive prenatal screening (NIPS) based on cell-free DNA (cfDNA) has provided millions of pregnant women with information about their risk for fetal chromosomal abnormalities. A primary driver of NIPS sensitivity for aneuploidy in a given maternal plasma sample is the fetal fraction (FF), which describes the proportion of cfDNA fragments that originate from the placenta. ${ }^{6}$ For most samples, FF values are between $4 \%$ and $30 \%{ }^{7}$ Many laboratories fail samples with $\mathrm{FF}<4 \%$ to diminish the risk of issuing false negative reports. Because the molecular and bioinformatic implementations of NIPS have evolved, diversified, and generally improved over time, sensitivity at progressively lower FF levels is platform- and laboratorydependent. ${ }^{6,8}$ Indeed, a recently published clinical experience study demonstrated that a customized whole-genome sequencing (WGS)-based NIPS, which does not fail low-FF samples, can have comparable accuracy at high FF and low FF for the common aneuploidies on chromosomes 13,18 , and $21 .^{9}$

Though the common aneuploidies have long been the main focus of NIPS because of their frequency and highly penetrant phenotype, clinically actionable chromosomal anomalies span a range of sizes and can occur anywhere in the genome. ${ }^{10-12}$ As such, a key frontier in NIPS development is to increase the resolution (i.e., detect smaller anomalies) and the scope (i.e., the number of regions) of the screen.

An example of increased resolution in NIPS is the screening for pathogenic microdeletion syndromes, ${ }^{10,13,14}$ such as DiGeorge syndrome ${ }^{15}$ and cri du chat syndrome; ${ }^{16}$ these arise from deletions of megabases of genome sequence, which are detectable to varying degrees on the primary NIPS platforms (i.e., WGS-based, ${ }^{17,18}$ single-nucleotide polymorphism (SNP)-based, ${ }^{19,20}$ and microarray-based ${ }^{21}$ platforms).

An example of increased scope in NIPS is screening for whole-chromosome aneuploidies on chromosomes other than 13,18 , and 21 , also referred to as rare autosomal aneuploidies (RAAs), which are associated with pregnancy complications ${ }^{12,22}$ and can now be discovered with WGSbased NIPS. ${ }^{12,17}$ Each NIPS platform has the potential to achieve higher resolution (e.g., via more WGS depth, more probed SNPs, or more microarray probes); however, because only the WGS-based approach to NIPS intrinsically interrogates the whole genome, it is manifestly better suited to increase the scope of screening than targeted approaches like array- and SNP-based NIPS.

\footnotetext{
${ }^{1}$ Myriad Genetics, Inc., Salt Lake City, UT, USA; ${ }^{2}$ Myriad Women’s Health, South San Francisco, CA, USA. Correspondence: Dale Muzzey (mwh_research@myriad.com)
}

These authors contributed equally: Dale Muzzey, Clement S. Chu 
WGS-based NIPS has recently been configured to identify novel microdeletions anywhere in the genome, though in the one peer-reviewed characterization of such an offering, novel deletions must exceed $7 \mathrm{MB}$ in length. ${ }^{17}$ Since many pathogenic microdeletions span $<7 \mathrm{MB}$, increasing copynumber sensitivity for small regions across the genome could have great clinical value. The resolution limit of genome-wide copy-number variant (gwCNV) detection is driven by the sequencing depth and the distribution of $\mathrm{FF}$ in the patient population (e.g., it is more challenging to detect small deletions in samples with low FF). Attempting to increase resolution via deeper sequencing provides diminishing returns and quickly yields an economically inviable screening test. Therefore, methods to increase the FF, if feasible, are preferable.

Though FF may seem an immutable and intrinsic feature of a cfDNA sample, it can be altered, and strategies for increasing $\mathrm{FF}$ are revealed by factors that correlate with FF. $^{6,23,24}$ For instance, $\mathrm{FF}$ is known to increase with gestational age, ${ }^{7}$ so drawing blood later in pregnancy leads to higher FF, though the effect is minor with FF increasing by $<1 \%$ per week. ${ }^{25,26} \mathrm{FF}$ also negatively correlates with firsttrimester body mass index (BMI) and maternal age, ${ }^{27}$ but these values are effectively constant for any given pregnancy. At the molecular level, it has been observed that fetal-derived cfDNA fragments tend to be shorter, ${ }^{28,29}$ hypermethylated, ${ }^{30-32}$ and enriched at different locations than maternal cfDNA fragments. ${ }^{33}$ Leveraging these biases at the molecular and bioinformatic levels has the potential to multiplicatively boost the FF of every sample.

Here we present an analytical validation and extended characterization of a fetal fraction amplification (FFA) technology that can be scalably applied to samples undergoing NIPS and yields significantly higher FF levels, thereby increasing sensitivity and specificity for all fetal anomalies arising from copy-number changes of any size across the genome.

\section{Ethics statement}

MATERIALS AND METHODS

All samples were from patients who had consented to deidentified research and received testing with the Prequel NIPS (Myriad Women's Health, South San Francisco, CA; as described ${ }^{9,34,35}$ ). The study was granted an institutional review board (IRB) exemption by Advarra (Pro00042194).

\section{FFA methodology}

The FFA technology leverages the reduced size of fetalderived cfDNA molecules-observed in several reports ${ }^{28,29}$ to increase the relative abundance of fetal cfDNA. Plasma was separated from a $10 \mathrm{~mL}$ whole-blood sample via centrifugation at $1600 \mathrm{~g}$ for 10 minutes. cfDNA was extracted from an aliquot of plasma using silanol-coated magnetic beads (Dynabeads, ThermoFisher) to yield approximately $5 \mathrm{ng}$ DNA per sample with an average fragment length of approximately $165 \mathrm{nt}$. cfDNA was quantified (PicoGreen,
ThermoFisher) and underwent conversion into a barcoded next-generation sequencing (NGS)-competent sequencing library suitable for the Illumina platform using manufacturer's instructions. Libraries were amplified via 12 rounds of polymerase chain reaction (PCR) (KAPA HiFi HotStart PCR Kit, Roche) before magnetic bead-based PCR cleanup. After another round of quantification, cfDNA libraries were size selected via gel electrophoresis on $2 \%$ agarose cassettes (BluePippin, Sage Science) following the manufacturer's instructions for "range" mode (a FFA workflow could include one of many different gel electrophoresis strategies, e.g., 2\% EGel EX from Invitrogen as in Qiao et al. ${ }^{28}$ and Liang et al. ${ }^{29}$ ). Short fragments were eluted from the gel until the average length of the eluted cfDNA was $140 \mathrm{nt}$, which preferentially retains fetal cfDNA and depletes maternal cfDNA (schematic in Fig. S1). The size-selected libraries had higher FF because fetal-derived fragments comprise a higher fraction of the total size-selected cfDNA. Consolidated libraries were sequenced on the Illumina HiSeq 4000 and processed via a custom bioinformatics pipeline characterized previously. ${ }^{35,36}$

\section{FFA validation}

The analytical validation of FFA involved 1264 patient samples from singleton pregnancies and 66 controls tested on 11 batches. Each patient sample was processed through two workflows: (1) standard WGS-based NIPS (i.e., Prequel without FFA) and (2) Prequel with FFA. The workflows were executed completely independently, each beginning with the extraction of cfDNA from replicate plasma aliquots. FF herein is measured either via a regression on autosomal bin depth or from the normalized depth of NGS data for a particular region (e.g., chrY, chr21).

Positive samples were sourced from our historical repository, prioritizing samples with confirmed clinical outcomes. The majority ( $81 \%$ of common aneuploidies and $55 \%$ overall) had orthogonally confirmed outcomes via diagnostic prenatal testing (e.g., amniocentesis or chorionic villus sampling) or diagnosis at birth. The minority without confirmed outcome were primarily screen-positive for microdeletions and RAAs on Prequel without FFA; thus, for these samples we are assessing comparability of Prequel with FFA to an already validated platform (i.e., Prequel without FFA). Negative samples were chosen randomly from the large population of patients who did not screen positive for any region of interest.

There were two types of negative-control samples used in validation and in every batch of samples screened in our clinical laboratory. First, to assess whether any contaminants were corrupting the steps in our DNA amplification, library preparation, and sequencing-batch-creation workflows, we included "no-template" controls (NTC) in which all steps were carried out as normal with the exception that no DNA was added to the DNA amplification. Next, to ensure that a euploid cfDNA sample indeed is identified as euploid by our pipeline, we included "pooled" controls. Pooled controls were either "XX" or "XY" and were created by pooling many 
hundreds of screen-negative samples with female or male fetuses, respectively.

Every batch of samples included in the validation contained NTCs and pooled controls, a plurality of negative samples, positives for each of the three common aneuploidies, and positives for some number of microdeletions, RAAs, and sex chromosome aneuploidies (SCAs). Some individual samples were populated more than once in a single batch or in different batches to assess the intra- and interbatch reproducibility, respectively.

To provide a more thorough characterization of FFA, some analyses herein augment the validation cohort with other samples tested internally during FFA development and verification. Only samples processed with the final, validated FFA protocol are included in such analyses. As is the case for all clinical samples tested in our laboratory, software-assisted manual review ${ }^{37}$ was performed on the samples tested in the verification and validation studies. Reviewers were blinded to the results from prior testing (e.g., via diagnostic outcome collection or prior sequencing analysis) when evaluating the results.

\section{Sensitivity and specificity assessment}

Because positive samples in analytical validation studies are relatively few in number and possibly unrepresentative (e.g., skewed toward above-average FF), using only the samples in the cohort to calculate sensitivity and specificity may not yield reflective estimates of clinical performance. Therefore, we developed a two-phase quantitative model that analyzes the positive samples included in the study but, importantly, overcomes the limitations of their rarity and potential biases. In the training phase of the model, a Markov chain Monte Carlo analysis deciphers how $z$-scores of samples aneuploid for a given region (e.g., chromosome 21) scale as a function of FF and read depth. In the simulation phase, the model generates $z$-score distributions for an arbitrarily large number of mock samples; importantly, the mock positives are now relatively unbiased in key features like FF and sufficiently numerous to power accurate and low-error estimates of sensitivity and specificity. By iterating over many different $z$ score thresholds (i.e., the $z$-score cutoff between a positive and negative screening result) and calculating the sensitivity and specificity among the mock positives and negatives, the model yields a receiver-operator characteristic (ROC) curve. The ROC model described above can be applied to a group of regions (e.g., microdeletions) or a particular region (e.g., 22q11.2).

The ROC model is a principled and clinically reflective method of calculating sensitivity and specificity to overcome biases and sample size limitations of a data set, but we also calculated these metrics with the standard approach (see Supplement). Fetal genotype calls with the FFA protocol were classified as true positive (TP), true negative (TN), false positive (FP), and false negative (FN) based on their concordance with confirmed outcome, where available. For each class of aneuploidy-common aneuploidies, RAAs,
SCAs, and microdeletions-sensitivity and specificity were calculated via standard definitions: sensitivity $=\mathrm{TP} /(\mathrm{TP}+$ $\mathrm{FN})$ and specificity $=\mathrm{TN} /(\mathrm{TN}+\mathrm{FP})$.

The ROC model was not developed to apply to aneuploidy calls that rely on multiple regions simultaneously, such as the SCAs that rely upon coupled information from chromosomes $\mathrm{X}$ and $\mathrm{Y}$; therefore, sensitivity and specificity values for SCAs were only calculated with the standard equations.

\section{Sex call accuracy measurement}

To determine the impact of FFA on distinguishing male and female fetuses, we calculated and compared the expected accuracy of sex calling for the standard NIPS and FFA protocols. Sex calling is based on the FF estimated from chrY $\left(\mathrm{FF}_{\mathrm{chrY}}\right)$, with female fetus pregnancies having $\mathrm{FF}_{\text {chrY }} \sim 0$ and male fetuses having $\mathrm{FF}_{\mathrm{chrY}}>0$. A normal distribution was fit to $\mathrm{FF}_{\mathrm{chr}}$ data from pregnancies called as having female fetuses, and a beta-distribution was fit to $\mathrm{FF}_{\mathrm{chr}}$ data from pregnancies called as having male fetuses. For a given sex-calling threshold, $y$, sex miscalls in female fetus pregnancies are estimated by the amount of the normal distribution fit with values exceeding $y$. Similarly, sex miscalls in male fetus pregnancies were calculated as the share of the beta-distribution fit with values less than $y$. On the assumption that males and females are equally likely, a value of $y$ was selected to minimize the total number of sex miscalls for both standard NIPS and FFA, and the difference in total expected FF-attributable sex miscalls was compared across protocols.

\section{RESULTS}

FFA increases FF an average of 2.3-fold for each sample

To directly measure the impact of FFA, we tested 2401 samples from our verification and validation studies with both the standard NIPS and FFA protocols, focusing particularly on the number of samples with $\mathrm{FF}<4 \%$, the threshold for low FF suggested by American College of Medical Genetics and Genomics (ACMG) ${ }^{38}$ As shown in Fig. 1 (top), 3.7\% of samples tested with the standard NIPS protocol had FF less than $4 \%$, whereas zero samples had low FF with the FFA protocol. The minimum FF observed in the FFA cohort was $4.9 \%$. As it has been observed that samples from patients with high BMI tend to have low FF and cause elevated test failures on several NIPS platforms, we partitioned samples by their BMI classes (Fig. 1, bottom). Critically, even at the highest BMI level (class III obesity), where $16 \%$ of samples had low FF with standard NIPS, every sample tested with the FFA protocol had $\mathrm{FF}>4 \%$ (minimum FF observed among class III patients was $7.1 \%$ ).

To confirm that FFA did not artifactually increase FF by corrupting our FF-inference regression model (see "Materials and methods"), we verified that the density of reads from chrY in pregnancies with male fetuses rose commensurately (Fig. S2). We conclude that FFA increases FF by directly increasing the relative abundance of fetal-derived cfDNA fragments in each sequenced sample. 


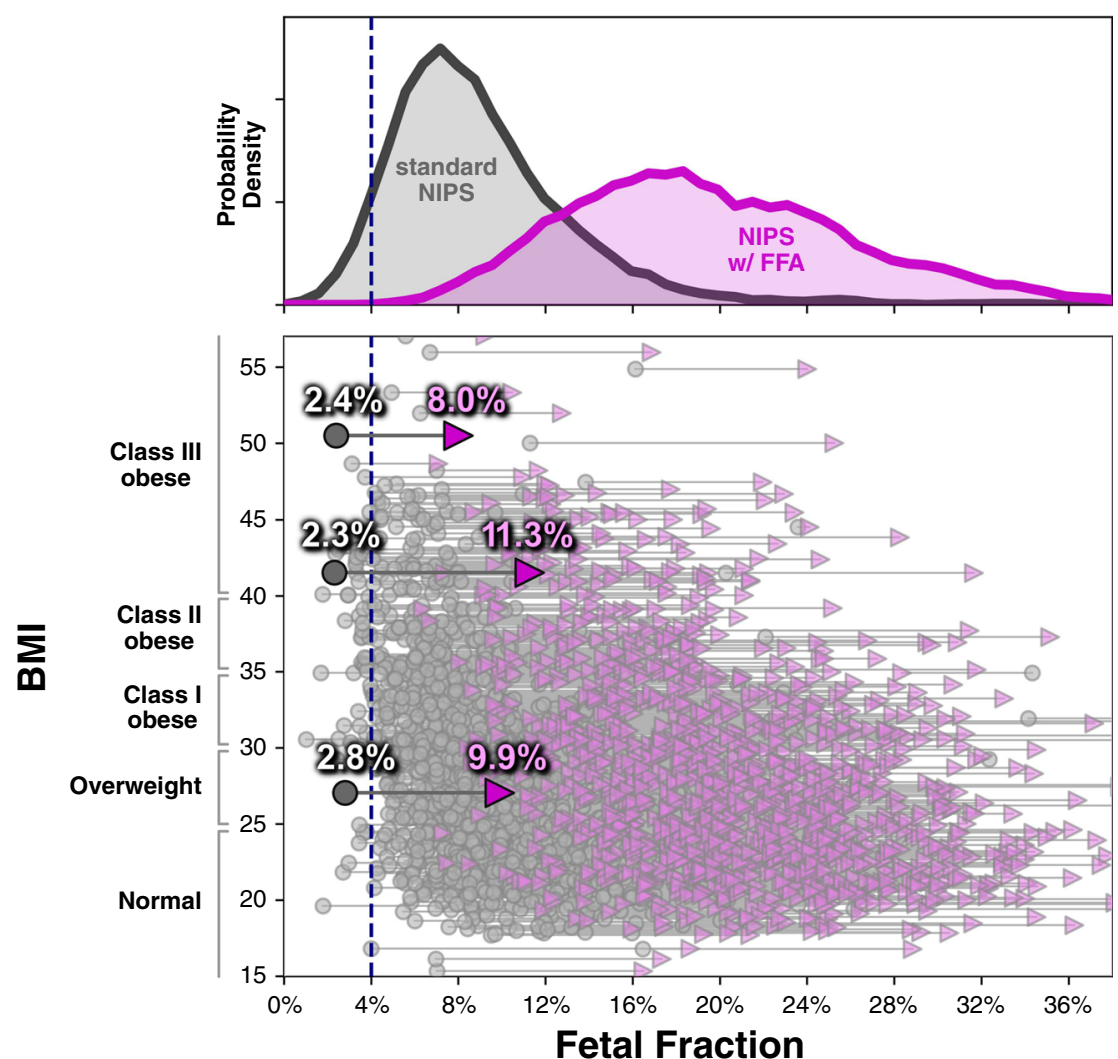

Fig. 1 Fetal fraction amplification (FFA) technology increases fetal fraction (FF) across all body mass index (BMI) levels. For 2401 patients who indicated BMI values on the test requisition form, the FF levels measured without FFA (gray circles) and with FFA (purple triangles) are plotted as a function of patients' BMI values (vertical axis). The top panel plots the histogram of samples with or without FFA. The dotted line is at $4 \% \mathrm{FF}$, the threshold below which the American College of Medical Genetics and Genomics (ACMG) considers a sample to have low FF. Three samples are highlighted as illustrative examples that had low FF before FFA but normal FF after FFA. NIPS noninvasive prenatal screening.

We examined sample-level changes in FF resulting from the FFA protocol because the upward shift in the overall FF distribution may obscure downward-shifting FF in a subset of samples. Figure 2 shows the relative gain in FF conferred by FFA. Notably, 2395 of the 2401 samples tested (99.8\%) had an increase in FF with FFA, with an average FF increase of 2.3fold. The relative sample-level gain in FF varied as a function of FF (Fig. 2): samples that were at low FF $(<4 \%)$ with standard NIPS had the largest FF gain, with an average of 3.9fold higher FF after undergoing FFA. Consistent with the FF gain diminishing at higher original FF levels, the six samples in which FF decreased with FFA had a median FF value of $27.8 \%$ (minimum $6.5 \%$ ), and the FF with FFA remained high (median: 25.4\%, minimum: 6.4\%).

\section{FFA increases NIPS sensitivity for all regions of interest}

In the same manner that $\mathrm{FF}$ can be directly measured in male fetus pregnancies from the relative NGS depth of chrX and chrY, it is possible to measure FF of aneuploid samples via the relative NGS depth on the aneuploid chromosome $\left(\mathrm{FF}_{\text {positive; }}\right.$ Fig. 3a). $\mathrm{FF}_{\text {positive }}$ is directly proportional to the $z$-score of an

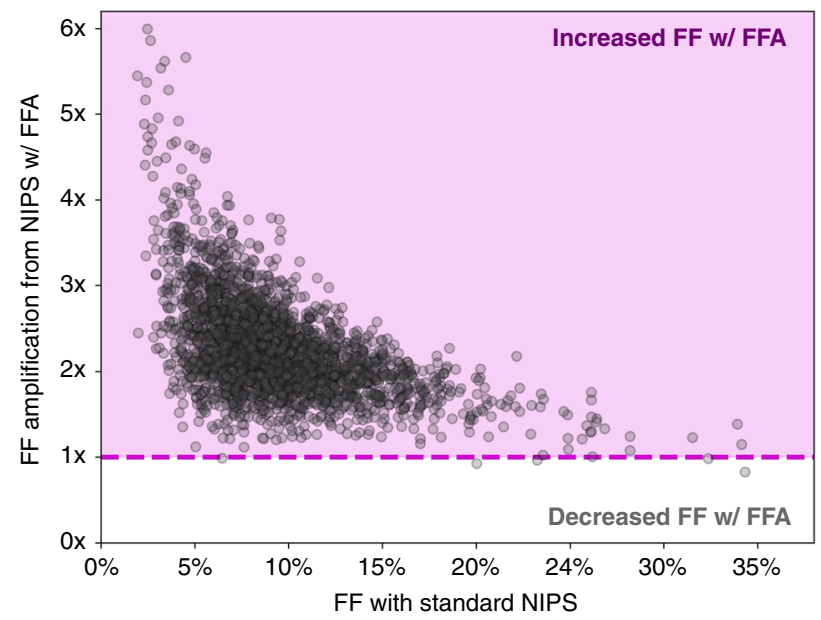

Fig. 2 Fetal fraction amplification (FFA) increases FF for $99.8 \%$ of samples tested and most appreciably for low-FF samples. The foldchange difference in FF as a result of applying FFA is plotted for individual samples as a function of the original FF without FFA. The dashed line indicates no change in FF, and samples in the purple-shaded region had increased FF with FFA. 
a

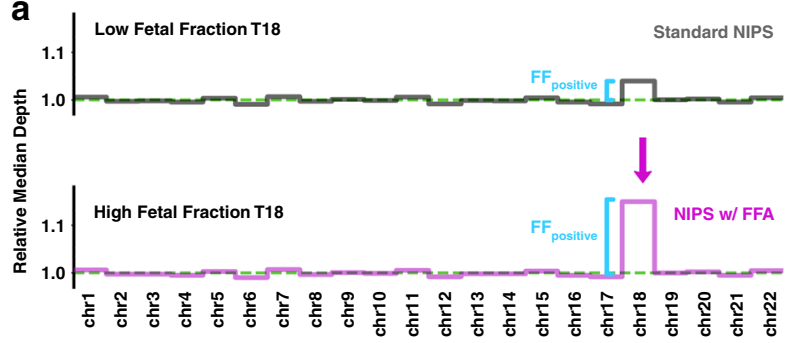

C
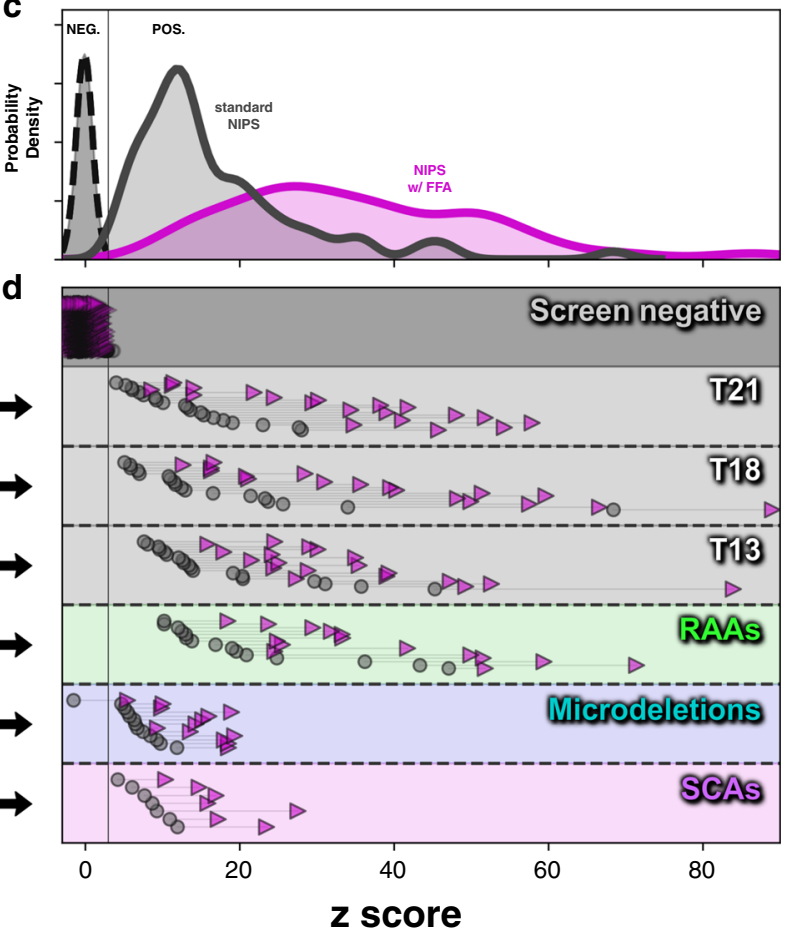

score b

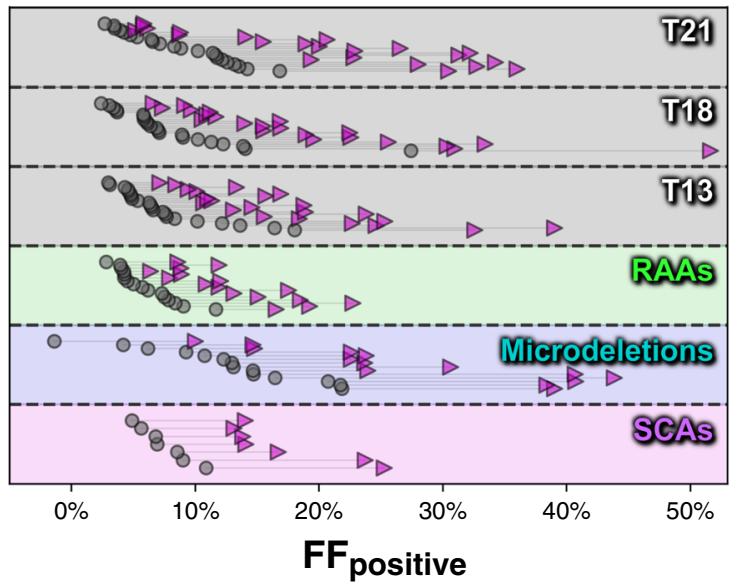

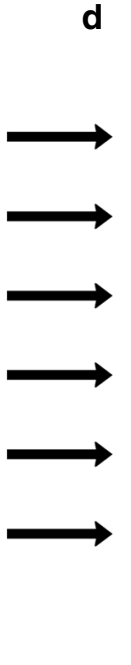

Fig. 3 Fetal fraction amplification (FFA) improves detection of fetal chromosome abnormalities by amplifying the signal of aneuploid regions while maintaining background noise. (a) Schematic of the change in median depth per autosome as a result of FFA. The extent of the deviation from

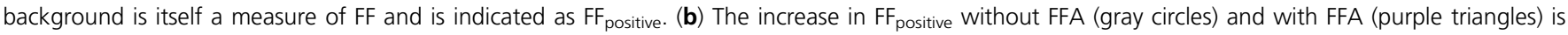
shown for aneuploid samples with the indicated chromosome anomalies. (c, d) z-scores without FFA (gray) and with FFA (purple) for the same samples as in (b) are stratified by their screening results and summarized either as population distributions (c) or as individual samples (d). For visual clarity in (c), the distribution of screen-negative samples (NEG.; dashed line) has been scaled to be of comparable height as the screen-positive distributions to the right (solid lines). The vertical solid line indicates the z-score cutoff between screen-negative (left) and screen-positive (right) results. For SCAs, only female fetus pregnancies are shown (i.e., MX and TX) because a $z$-score is used to identify chrX aneuploidies, whereas a two-dimensional analysis that does not use $z$ scores (not shown) is required for identification of XXY and XYY ( $F_{\text {positive }}$ increased in all XXY and XYY pregnancies tested with FFA). NIPS noninvasive prenatal screening, RAA rare autosomal aneuploidies, SCA sex chromosome aneuploidies.

aneuploid region, and a higher $z$-score means that aneuploidy is more likely to be detected. Therefore, if FFA increases $\mathrm{FF}_{\text {positive }}$ of aneuploid regions, then FFA also increases NIPS sensitivity.

In every positive sample tested-across common aneuploidies, RAAs, and microdeletions-FFA yielded an increase in $\mathrm{FF}_{\text {positive }}$ (Fig. 3b). As expected, FFA also increased $z$-scores for every tested aneuploid sample, whereas the $z$-score distribution for euploid samples was unchanged (Fig. 3c, d). Larger $z$-score separation between positive and negative samples heightens the ability to discriminate such samples and thereby lessens the chances of false negatives and false positives. Together, these observations demonstrate that FFA directly increases the concentration of fetal-derived reads in each sample and enhances the sensitivity and specificity of NIPS.

A sample that screened negative for the $5 p$ microdeletion with standard NIPS but positive with FFA (Figs. S3, 3b, d) provided further support for the enhanced sensitivity for fetal chromosome abnormalities that FFA confers. For this 3-MB microdeletion, the copy-number change was conspicuously apparent in the FFA data (Fig. S3), converting a $z$-score below
Table 1 Analytical performance metrics as estimated from ROC analysis.

\begin{tabular}{lll} 
& $\begin{array}{l}\text { Analytical } \\
\text { sensitivity }\end{array}$ & $\begin{array}{l}\text { Analytical } \\
\text { specificity }\end{array}$ \\
\hline $\begin{array}{l}\text { Common aneuploidies } \\
\text { (aggregate) }\end{array}$ & $99.988 \% \pm 0.004 \%$ & $99.968 \% \pm 0.005 \%$ \\
\hline T21 & $99.990 \% \pm 0.005 \%$ & $99.996 \% \pm 0.001 \%$ \\
\hline T18 & $99.990 \% \pm 0.002 \%$ & $99.996 \% \pm 0.001 \%$ \\
\hline T13 & $99.978 \% \pm 0.005 \%$ & $99.976 \% \pm 0.005 \%$ \\
\hline RAAs (aggregate) & $99.695 \% \pm 0.305 \%$ & $99.981 \% \pm 0.010 \%$ \\
\hline Microdeletions (aggregate) & $97.172 \% \pm 0.054 \%$ & $99.767 \% \pm 0.012 \%$ \\
\hline $\begin{array}{l}\text { DiGeorge syndrome } \\
\text { (22q11.2) }\end{array}$ & $95.633 \% \pm 0.071 \%$ & $99.949 \% \pm 0.005 \%$ \\
\hline
\end{tabular}

$R A A$ rare autosomal aneuploidies, $R O C$ receiver-operator characteristic.

the calling threshold into one above the threshold (Fig. 3d, microdeletions track).

To quantify the gain in sensitivity and specificity achievable with FFA, we analyzed the relationship between various clinical and technical metrics, such as $z$-scores, depth, incidence, and FF (see "Materials and methods"). The ROC curves (Fig. S4) for different classes of chromosomal 
abnormalities show that FFA enables near-perfect analytical sensitivity with near-perfect analytical specificity (Table 1). The sensitivity of common aneuploidies-shown to be high in our clinical experience without $\mathrm{FFA}^{9}$-is marginally higher with FFA, as is the aggregate sensitivity of RAAs (Fig. S4). However, the gain in microdeletion sensitivity is substantial: with FFA, the aggregate sensitivity for five common microdeletions is $97.2 \%$ at a joint specificity of $99.8 \%$. For DiGeorge syndrome in particular, FFA has an expected analytical sensitivity of $95.6 \%$ with an analytical specificity of 99.95\%.

In addition to assessing performance with the ROC analysis above, we also observed that all samples with a confirmed aneuploidy or microdeletion were correctly identified with FFA (Table S1). Finally, the results were repeatable and reproducible within and across batches, respectively (Tables S2, S3). Together, these experiments establish the analytical validity of FFA.

FFA increases sex-calling accuracy relative to standard NIPS Sex miscalls in NIPS arise from limitations that are either biological (e.g., true fetal mosaicism, vanishing twin) or technical (e.g., low FF). The former are an unavoidable aspect of NIPS on any screening platform (many sex miscalls occur at FF far greater than $4 \%$ ), but the latter could be mitigated by FFA due to its ability to increase the FF of all samples and thereby remove borderline calls. Figure 4 shows distributions

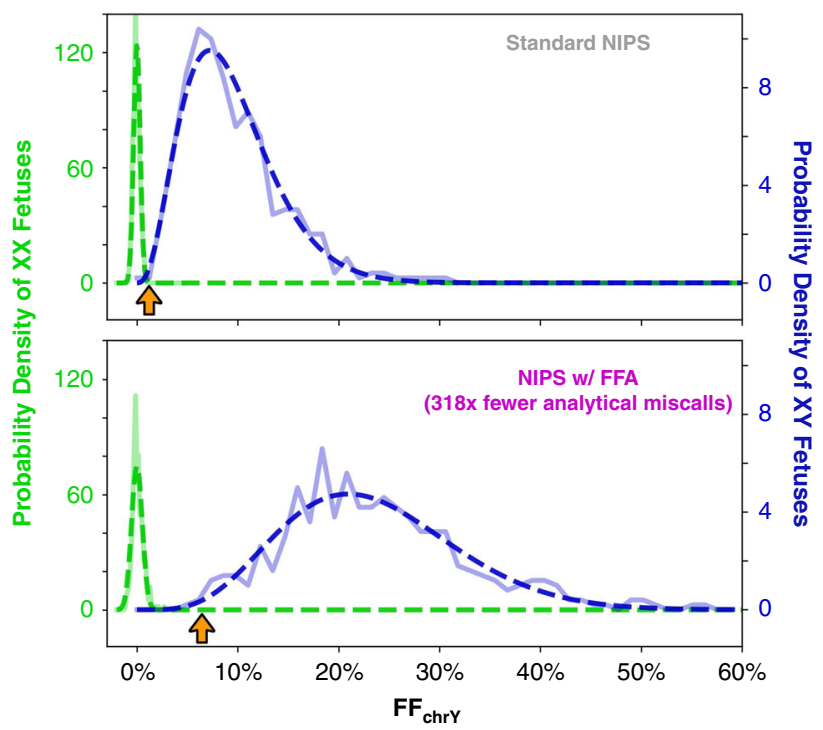

Fig. 4 Fetal fraction amplification (FFA) increases the analytical accuracy of fetal sex calling. For both standard noninvasive prenatal screening (NIPS) (top) and Prequel with FFA (bottom), the distribution of $\mathrm{FF}_{\text {chry }}$ values is shown for samples called as female (green) or male (blue). Solid lines indicate raw data, and the dashed lines show best-fit traces for the female (Gaussian) and male (beta) populations. Only euploid samples are included in the analysis. The orange arrow depicts one sample tested on both platforms, called female in standard NIPS and male with FFA (the fetus was confirmed to be male). After minimizing the number of estimated miscalls on each platform (see "Materials and methods"), analytical miscalls are predicted to drop 318-fold with FFA. of $\mathrm{FF}_{\mathrm{chr}}$ (i.e., the FF as measured from the NGS read density on chromosome $\mathrm{Y}$ ) for male fetus and female fetus pregnancies as observed for standard NIPS and FFA. Notably, the separation between male and female $\mathrm{FF}_{\mathrm{chr}}$ distributions is larger with FFA, reducing the chance of sex miscalls due to borderline $\mathrm{FF}_{\mathrm{chr}}$ values by an estimated $318 \times$ (see "Materials and methods"). Underscoring the improvement, one sample tested in the validation study (Fig. 4, orange arrow) was borderline in standard NIPS and miscalled as XX; however, the sample was clearly XY upon screening with FFA, and the pregnancy was orthogonally confirmed via ultrasound to be male.

\section{DISCUSSION}

Here we validated and characterized the performance of a NIPS that applies FFA technology to every sample. For $99.8 \%$ of samples tested, FF increased with FFA, with the average gain being 2.3-fold. Low-FF samples received the largest FF scaling, and of 2401 samples tested, 3.7\% had low FF before FFA, but none had low FF after FFA. Importantly, the gain in FF is molecular and not algorithmic: FFA distinguishes between maternal and fetal DNA, and it increases the relative proportion of fetal DNA in the sample undergoing WGS. Though the combination of our custom algorithm and WGS technology showed high sensitivity and specificity for common aneuploidies across the FF spectrum without FFA technology, ${ }^{9}$ application of FFA increases performance for each type of aneuploidy, with the gain being particularly substantial for microdeletions.

The literature is replete with reports and professional society statements expressing concern about low-FF samples. ${ }^{8,38,39}$ Many publications have explored and debated the merits of different approaches to handling low-FF samples: optimizing NIPS algorithms to issue confident results at low $\mathrm{FF},{ }^{9}$ failing such samples entirely, or pursuing mitigation strategies for failed low-FF samples, such as sequential redraw ${ }^{40,41}$ and FF-based risk scores. ${ }^{42,43}$ However, consensus has remained elusive. As such, the FFA technology represents an advance in NIPS because samples that would have had low FF on standard NIPS are molecularly transformed into samples that have high FF. Accompanying the increase in test performance that FFA affords, we anticipate that this assay improvement will increase the confidence that providers and patients have in their results with NIPS.

FFA has a dramatic impact on the performance of microdeletion screening in NIPS. For common microdeletions, the expected aggregate sensitivity increases (Table 1, Fig. S4), reaching $97.2 \%$ with FFA. In the past, when microdeletion sensitivity and specificity were low for microdeletions, the American College of Obstetricians and Gynecologists (ACOG) recommended against microdeletion screening; however, we expect that sensitivity $>97 \%$ and specificity $>99 \%$ for microdeletions could allow professional societies to consider the clinical merits rather than the technological limitations of screening for microdeletions. 
Beyond the common microdeletions, our data suggest that FFA will increase the resolution of gwCNV detection, enabling confident identification of microdeletions below the current limit of $7 \mathrm{MB}$ achievable with standard NIPS. Short microdeletions in samples with low FF can be challenging to detect with NIPS and limit sensitivity, but FFA raises the achievable sensitivity limit by reducing the frequency of low-FF samples. Notably, the 22q11.2 microdeletion, which causes DiGeorge syndrome, most commonly spans $\sim 2-3 \mathrm{MB}$ and has an expected sensitivity of $95.6 \%$ with FFA. To ensure that false positives are rare, the resolution limit for novel gwCNV detection may need to be above $3 \mathrm{MB}$, but dbVar contains more than a thousand unique pathogenic microdeletions between $3 \mathrm{MB}$ and $7 \mathrm{MB}$ in size, a number of which are associated with clinically serious phenotypes, so any gains in resolution should increase the utility of NIPS for patients and providers.

Even if two NIPS laboratories were to test the same plasma sample, the reported FF and sensitivity for aneuploidy may differ due to variations in the laboratories' respective molecular and computational protocols. For instance, based on differing methods of aligning, filtering, counting, and analyzing NGS reads, a laboratory reporting $8 \%$ FF could have higher aneuploidy sensitivity than a laboratory reporting $10 \%$ FF. These differences complicate interlab comparisons of NIPS performance, especially since laboratories demonstrate performance on different sample sets and with different study designs (e.g., clinical experience study vs. analytical validation study). As such, it can be difficult to make conclusive statements about relative NIPS performance. However, here we have demonstrated an unequivocal NIPS performance gain: two protocols (standard NIPS and FFA) were compared on a single set of samples within a single laboratory using a single aneuploidy-calling algorithm. FF increased 2.3-fold on average, and this FF increase resulted from a higher frequency of fetal-derived NGS reads. Beyond showing evidence for a relative gain in performance, the ROC analysis we performed yields an estimate of analytical sensitivity and specificity in an unbiased cohort reflective of a large population of clinical samples.

The FFA strategy described herein increases the FF of a sample at the molecular level via size selection upstream of sequencing, yet it is also possible to increase FF via algorithmic size selection downstream of sequencing. Specifically, the bioinformatics pipeline could calculate each fragment's length based on the respective mapping positions of its paired-end reads and upweight shorter fragments in the analysis. However, the disadvantage of this bioinformatic approach is that substantial resources would still be consumed by sequencing longer fragments-likely to be maternal-derived-that contribute little to fetal aneuploidy detection. By contrast, when performing molecular size selection upstream of sequencing, all of the sequenced fragments have elevated likelihood of being fetal-derived.
In conclusion, FFA renders a FF cutoff obsolete and negates the tradeoff often needed for a low test failure rate to coexist with highly accurate results. Debates about how best to serve patients with low-FF plasma samples can now be relegated to the past because low-FF samples need not exist.

\section{SUPPLEMENTARY INFORMATION}

The online version of this article (https://doi.org/10.1038/s41436020-01009-5) contains supplementary material, which is available to authorized users.

\section{ACKNOWLEDGEMENTS}

The authors are grateful to Susan Hancock, Katherine Johansen Taber, and Anna Gardiner for assistance with the manuscript. Also, the authors acknowledge the support of the Myriad clinical laboratory in South San Francisco for processing the validation samples.

\section{DISCLOSURE}

All authors are current or former employees and equity holders of Myriad Genetics.

Publisher's note Springer Nature remains neutral with regard to jurisdictional claims in published maps and institutional affiliations.

\section{REFERENCES}

1. Chiu RW, Chan KC, Gao Y, et al. Noninvasive prenatal diagnosis of fetal chromosomal aneuploidy by massively parallel genomic sequencing of DNA in maternal plasma. Proc Natl Acad Sci USA. 2008;105: 20458-20463.

2. Fan HC, Blumenfeld YJ, Chitkara U, Hudgins L, Quake SR. Noninvasive diagnosis of fetal aneuploidy by shotgun sequencing DNA from maternal blood. Proc Natl Acad Sci USA. 2008;105:16266-16271.

3. Chiu RW, Akolekar R, Zheng YW, et al. Non-invasive prenatal assessment of trisomy 21 by multiplexed maternal plasma DNA sequencing: large scale validity study. BMJ. 2011;342:c7401.

4. Palomaki GE, Kloza EM, Lambert-Messerlian GM, et al. DNA sequencing of maternal plasma to detect Down syndrome: an international clinical validation study. Genet Med. 2011;13:913-920.

5. Srinivasan A, Bianchi DW, Huang $H$, Sehnert AJ, Rava RP. Noninvasive detection of fetal subchromosome abnormalities via deep sequencing of maternal plasma. Am J Hum Genet. 2013;92:167-176.

6. Hui L, Bianchi DW. Fetal fraction and noninvasive prenatal testing: What clinicians need to know. Prenat Diagn. 2020;40:155-163.

7. Wang E, Batey A, Struble C, Musci T, Song K, Oliphant A. Gestational age and maternal weight effects on fetal cell-free DNA in maternal plasma. Prenat Diagn. 2013;33:662-666.

8. Artieri CG, Haverty C, Evans EA, et al. Noninvasive prenatal screening at low fetal fraction: comparing whole-genome sequencing and singlenucleotide polymorphism methods. Prenat Diagn. 2017;37:482-490.

9. Hancock S, Ben-Shachar R, Adusei $C$, et al. Clinical experience across the fetal-fraction spectrum for a non-invasive prenatal screen with low testfailure rate. Ultrasound Obstet Gynecol. 2020;56:422-430.

10. Wapner RJ, Babiarz JE, Levy B, et al. Expanding the scope of noninvasive prenatal testing: detection of fetal microdeletion syndromes. Am J Obstet Gynecol. 2015;212:332 e331-e339.

11. Advani HV, Barrett AN, Evans MI, Choolani M. Challenges in non-invasive prenatal screening for sub-chromosomal copy number variations using cell-free DNA. Prenat Diagn. 2017;37:1067-1075.

12. Pertile MD, Halks-Miller M, Flowers N, et al. Rare autosomal trisomies, revealed by maternal plasma DNA sequencing, suggest increased risk of feto-placental disease. Sci Transl Med. 2017;9:eaan1240.

13. Chen Y, Yu Q, Mao X, Lei W, He M, Lu W. Noninvasive prenatal testing for chromosome aneuploidies and subchromosomal microdeletions/ 
microduplications in a cohort of 42,910 single pregnancies with different clinical features. Hum Genomics. 2019;13:60.

14. Liang D, Cram DS, Tan H, et al. Clinical utility of noninvasive prenatal screening for expanded chromosome disease syndromes. Genet Med. 2019;21:1998-2006.

15. Grati FR, Gross SJ. Noninvasive screening by cell-free DNA for $22 q 11.2$ deletion: benefits, limitations, and challenges. Prenat Diagn. 2019;39: 70-80.

16. Cerruti Mainardi P. Cri du chat syndrome. Orphanet J Rare Dis. 2006;1:33

17. Lefkowitz RB, Tynan JA, Liu T, et al. Clinical validation of a noninvasive prenatal test for genomewide detection of fetal copy number variants. Am J Obstet Gynecol. 2016;215:227 e1-227 e16.

18. Yu D, Zhang $K$, Han $M$, et al. Noninvasive prenatal testing for fetal subchromosomal copy number variations and chromosomal aneuploidy by low-pass whole-genome sequencing. Mol Genet Genomic Med. 2019;7:e674.

19. Ravi $H$, McNeill G, Goel S, et al. Validation of a SNP-based non-invasive prenatal test to detect the fetal $22 q 11.2$ deletion in maternal plasma samples. PLOS ONE. 2018;13:e0193476.

20. Hui L, Cell-free DNA. testing for 22q11.2 deletion syndrome: appraising the viability, effectiveness and appropriateness of screening. Ultrasound Obstet Gynecol. 2016;47:137-141.

21. Schmid M, Wang E, Bogard PE, et al. Prenatal screening for $22 q 11.2$ deletion using a targeted microarray-based cell-free DNA test. Fetal Diagn Ther. 2018;44:299-304.

22. Van Opstal D, van Maarle MC, Lichtenbelt K, et al. Origin and clinical relevance of chromosomal aberrations other than the common trisomies detected by genome-wide NIPS: results of the TRIDENT study. Genet Med. 2018;20:480-485.

23. Peng $X L$, Jiang $P$. Bioinformatics approaches for fetal DNA fraction estimation in noninvasive prenatal testing. Int J Mol Sci. 2017:18:453.

24. Hestand MS, Bessem M, van Rijn P, et al. Fetal fraction evaluation in noninvasive prenatal screening (NIPS). Eur J Hum Genet. 2019;27:198-202.

25. Livergood MC, LeChien KA, Trudell AS. Obesity and cell-free DNA "no calls": is there an optimal gestational age at time of sampling? Am J Obstet Gynecol. 2017;216:413 e411-413 e419.

26. Yared E, Dinsmoor MJ, Endres LK, et al. Obesity increases the risk of failure of noninvasive prenatal screening regardless of gestational age. Am J Obstet Gynecol. 2016;215:370 e1-376 e6.

27. Suzumori $N$, Ebara $T$, Yamada $T$, et al. Fetal cell-free DNA fraction in maternal plasma is affected by fetal trisomy. J Hum Genet. 2016;61: 647-652.

28. Qiao $L, Y u B$, Liang $Y$, et al. Sequencing shorter cfDNA fragments improves the fetal DNA fraction in noninvasive prenatal testing. Am J Obstet Gynecol. 2019;221:345 e1-345 e11.

29. Liang $\mathrm{B}$, Li H, He Q, et al. Enrichment of the fetal fraction in non-invasive prenatal screening reduces maternal background interference. Sci Rep. 2018;8:17675.

30. Sun K, Jiang P, Chan KC, et al. Plasma DNA tissue mapping by genomewide methylation sequencing for noninvasive prenatal, cancer, and transplantation assessments. Proc Natl Acad Sci USA. 2015;112: E5503-E5512.

31. Nygren AO, Dean J, Jensen TJ, et al. Quantification of fetal DNA by use of methylation-based DNA discrimination. Clin Chem. 2010;56:1627-1635.

32. Lun FM, Chiu RW, Sun K, et al. Noninvasive prenatal methylomic analysis by genomewide bisulfite sequencing of maternal plasma DNA. Clin Chem. 2013;59:1583-1594.
33. Chan $K C$, Jiang $P$, Sun $K$, et al. Second generation noninvasive fetal genome analysis reveals de novo mutations, single-base parental inheritance, and preferred DNA ends. Proc Natl Acad Sci USA. 2016;113:E8159-E8168.

34. Kaseniit KE, Hogan GJ, D'Auria KM, Haverty C, Muzzey D. Strategies to minimize false positives and interpret novel microdeletions based on maternal copy-number variants in 87,000 noninvasive prenatal screens. BMC Med Genomics. 2018:11:90.

35. Muzzey D, Goldberg JD, Haverty C. Noninvasive prenatal screening for patients with high body mass index: evaluating the impact of a customized whole-genome sequencing workflow on sensitivity and residual risk. Prenat Diagn. 2020;40:333-341.

36. Muzzey D. The technology and bioinformatics of cell-free DNA based NIPT. London: Elsevier; 2018

37. Muzzey D, Kash S, Johnson Jl, et al. Software-assisted manual review of clinical next-generation sequencing data: an alternative to routine Sanger sequencing confirmation with equivalent results in $>15,000$ germline DNA screens. J Mol Diagn. 2019;21:296-306.

38. Gregg AR, Skotko BG, Benkendorf $\mathrm{JL}$, et al. Noninvasive prenatal screening for fetal aneuploidy, 2016 update: a position statement of the American College of Medical Genetics and Genomics. Genet Med. 2016;18:1056-1065.

39. Committee on Practice Bulletins-Obstetrics, Committee on Genetics, Society for Maternal-Fetal Medicine. Practice bulletin no. 163: screening for fetal aneuploidy. Obstet Gynecol. 2016;127:e123-e137.

40. Benn P, Valenti E, Shah S, Martin K, Demko Z. Factors associated with informative redraw after an initial no result in noninvasive prenatal testing. Obstet Gynecol. 2018;132:428-435.

41. Ryan A, Hunkapiller $N$, Banjevic $M$, et al. Validation of an enhanced version of a single-nucleotide polymorphism-based noninvasive prenatal test for detection of fetal aneuploidies. Fetal Diagn Ther. 2016;40: 219-223.

42. McKanna T, Ryan A, Krinshpun S, et al. Fetal fraction-based risk algorithm for non-invasive prenatal testing: screening for trisomies 13 and 18 and triploidy in women with low cell-free fetal DNA. Ultrasound Obstet Gynecol. 2019;53:73-79.

43. Benn P, Martin K, McKanna T, Valenti E, Billings P, Demko Z. Combining the use of a fetal fraction-based risk algorithm and probability of an informative redraw in noninvasive prenatal testing for fetal aneuploidy. J Genet Couns. 2019;29:800-806.

\section{(i)}

Open Access This article is licensed under a Creative Commons Attribution 4.0 International License, which permits use, sharing, adaptation, distribution and reproduction in any medium or format, as long as you give appropriate credit to the original author(s) and the source, provide a link to the Creative Commons license, and indicate if changes were made. The images or other third party material in this article are included in the article's Creative Commons license, unless indicated otherwise in a credit line to the material. If material is not included in the article's Creative Commons license and your intended use is not permitted by statutory regulation or exceeds the permitted use, you will need to obtain permission directly from the copyright holder. To view a copy of this license, visit http://creativecommons.org/licenses/ by/4.0/.

(C) The Author(s) 2020 